\title{
NOTE ON RELATIONS CONNECTING CERTAIN CASES OF CONVERGENCE IN THE MEAN*
}

\section{BY DUNHAM JACKSON}

1. Introduction. A lemma from which various inferences can be drawn with regard to the convergence of sequences of trigonometric sums reads as follows. $\dagger$

LEMMA A. If $f(x)$ is a continuous function of period $2 \pi, T_{n}(x)$ a trigonometric sum of the nth order, and

$$
G_{n s}=\int_{-\pi}^{\pi}\left|f(x)-T_{n}(x)\right|^{s} d x
$$

and if there exists a trigonometric sum $t_{n}(x)$ of the nth order such that $\left|f(x)-t_{n}(x)\right| \leqq \epsilon_{n}$ everywhere, then

$$
\left|f(x)-T_{n}(x)\right| \leqq 4\left(n G_{n s}\right)^{1 / s}+5 \epsilon_{n}
$$

for all values of $x$.

The exponent $s$ may be any positive constant. In view of the continuity of $f(x)$, it is possible to construct approximating sums $t_{n}(x)$ for successive values of $n$ and to assign corresponding upper bounds $\epsilon_{n}$ for the error of the approximation so that $\lim _{n \rightarrow \infty} \epsilon_{n}=0$. It follows as an immediate corollary of the lemma that if a sequence of sums $T_{n}(x)$ has the property that $\lim _{n \rightarrow \infty} n G_{n s}=0$, for fixed $s$, then $T_{n}(x)$ converges uniformly toward $f(x)$ as $n$ becomes infinite. This may be regarded as constituting a relationship between the convergence properties of two measures of the discrepancy between $f(x)$ and the sum $T_{n}(x)$, regarded as an approximation to $f(x)$ : the mean value $G_{n s} /(2 \pi)$ of the $s$ th power of the error, and the maximum value attained by the error at any single point. The latter will converge to zero if the former approaches zero with sufficient rapidity. On the other hand, if the maximum error approaches zero, the mean will necessarily approach zero, without further restriction.

* Presented to the Society, December 27, 1933.

$\dagger$ See D. Jackson, Certain problems of closest approximation, this Bulletin vol. 39 (1933), pp. 889-908, Lemma 5. 
The aim of this paper is to point out corresponding relations between the properties of convergence of the means corresponding to two different exponents $s$ and $\sigma$. One side of the relationship is an obvious consequence of Hölder's inequality; the other is recognizable as a simple corollary of the lemma. As would naturally be expected, the result corresponding to the use of the maximum error as measure of the discrepancy is approached as a limit if $\sigma$ is allowed to become infinite. For the purpose in hand the lemma as first stated will be replaced by a slightly modified version, which is not effective for the proof of uniform convergence but admits consideration of discontinuous functions in the treatment of convergence in the mean. It may be emphasized that except when expressly noted below no minimizing property need be ascribed to the sums $T_{n}(x)$; they are not necessarily determined by any criterion of closest approximation.

To the discussion of trigonometric approximation there corresponds a parallel treatment of approximation by means of polynomials, which will, however, not be carried through in detail.

The principle is illustrated in exceedingly simple form by a problem which may be regarded as a special case of the preceding for $f(x) \equiv 0$, but is more directly of interest for its own sake. This special problem will be treated first.

2. Integrals of Powers of Trigonometric Sums and Polynomials. If $\phi(x)$ is a non-negative function over an interval $(a, b)$, and if the value of the integral

$$
H_{s}=\int_{a}^{b}[\phi(x)]^{s} d x
$$

is known, an upper bound for the magnitude of the integral $H_{\sigma}$ with a value of $\sigma<s$ is given by Hölder's inequality. For $\sigma>s$ no such upper bound is in general available. For special classes of functions $\phi(x)$, however, a relation of inequality giving an upper bound is obtainable in this case also.

Let $T_{n}(x)$ be a trigonometric sum of the $n$th order, and let

$$
H_{n s}=\int_{-\pi}^{\pi}\left|T_{n}(x)\right|^{s} d x
$$

with an arbitrary positive exponent $s$. For $\sigma<s$, by Hölder's 
inequality, $H_{n} \leqq(2 \pi)^{1-(\sigma / s)}\left(H_{n s}\right)^{(\sigma / s)}$. The following lemma, the proof of which* (like that of Lemma A) depends on Bernstein's theorem on the derivative of a trigonometric sum, leads at once to the desired inequality in the contrary case.

LEMmA B. If $T_{n}(x)$ is a trigonometric sum of the nth order, if

$$
H_{n s}=\int_{-\pi}^{\pi}\left|T_{n}(x)\right|^{8} d x
$$

and if $\lambda_{n}$ is the maximum of $\left|T_{n}(x)\right|$, then $\mu_{n} \leqq 2\left(n H_{n s}\right)^{1 / 8}$.

If $\sigma>s$, application of this lemma gives

$$
\begin{aligned}
H_{n \sigma} & =\int_{-\pi}^{\pi}\left|T_{n}(x)\right|^{\sigma-s}\left|T_{n}(x)\right|^{s} d x \\
& \leqq\left[2\left(n H_{n s}\right)^{1 / s}\right]^{\sigma-s} H_{n s}=2^{\sigma-s} n^{(\sigma / s)-1}\left(H_{n s}\right)^{\sigma / s} .
\end{aligned}
$$

The result involves the order of the trigonometric sum through the factor $n^{(\sigma / s)-1}$. The inequality can be written in the more symmetrical form

$$
\left(n H_{n \sigma}\right)^{1 / \sigma} \leqq 2^{1-(s / \sigma)}\left(n H_{n s}\right)^{1 / s} .
$$

Similarly, if $P_{n}(x)$ is a polynomial of the $n$th degree, and

$$
H_{n s}=\int_{a}^{b}\left|P_{n}(x)\right|^{s} d x
$$

then

$$
H_{n \sigma} \leqq(b-a)^{1-(\sigma / s)}\left(H_{n s}\right)^{\sigma / s}
$$

for $\sigma<s$, by Hölder's inequality, and

$$
H_{n \sigma} \leqq C^{\sigma} n^{2(\sigma-s) / s}\left(H_{n s}\right)^{\sigma / s}, \quad\left(n^{2} H_{n \sigma}\right)^{1 / \sigma} \leqq C\left(n^{2} H_{n s}\right)^{1 / s},
$$

for $\sigma>s$, in consequence of a proposition $\dagger$ analogous to Lemma $\mathrm{B}$, the constant $C$ having the value $\left\{2[4 /(b-a)]^{1 / s}\right\}^{(\sigma-s) / \sigma}$.

3. Convergence of Trigonometric Approximation. Let $f(x)$ be a given function of period $2 \pi$ which is bounded and integrable (in the sense of Riemann or in the sense of Lebesgue), and let $T_{n}(x)$ be a trigonometric sum of the $n$th order. Let

\footnotetext{
* See this Bulletin, loc. cit., Lemma 1.
}

† See this Bulletin, loc. cit., Lemma 2. 


$$
G_{n s}=\int_{-\pi}^{\pi}\left|f(x)-T_{n}(x)\right| \cdot d x
$$

with any value of $s>0$. If $\sigma<s$, application of Hölder's inequality gives

$$
G_{n \sigma} \leqq(2 \pi)^{1-(\sigma / s)}\left(G_{n s}\right)^{\sigma / s} .
$$

It follows that if a sequence of sums $T_{n}(x)$ is given so that $\lim _{n \rightarrow \infty} G_{n s}=0$ for a fixed value of $s$, then $\lim _{n \rightarrow \infty} G_{n \sigma}=0$ for any fixed $\sigma<s$.

For treatment of the case $\sigma>s$ the following modification of Lemma A will be established.

LEMmA C. If $f(x)$ is a bounded and integrable function of period $2 \pi$ having $M$ as an upper bound for its absolute value, $T_{n}(x)$ a trigonometric sum of the nth order, and

then

$$
G_{n s}=\int_{-\pi}^{\pi}\left|f(x)-T_{n}(x)\right| s d x
$$

$$
\left|f(x)-T_{n}(x)\right| \leqq 4\left(n G_{n s}\right)^{1 / s}+5 M
$$

for all values of $x$.

Let $\mu_{n}$ be the maximum of $\left|T_{n}(x)\right|$, attained for $x=x_{0}$. By Bernstein's theorem, $\left|T_{n}^{\prime}(x)\right| \leqq n \mu_{n}$ everywhere. For $\left|x-x_{0}\right|$ $\leqq 1 /(2 n), \quad\left|T_{n}\left(x_{0}\right)-T_{n}(x)\right| \leqq \mu_{n} / 2$, and $\left|T_{n}(x)\right| \geqq \mu_{n} / 2$. If $\mu_{n} \geqq 4 M$, so that $|f(x)| \leqq \mu_{n} / 4$, then $\left|f(x)-T_{n}(x)\right| \geqq \mu_{n} / 4$ throughout the specified range of values for $x$. The length of the interval being $1 / n$,

$$
G_{n s} \geqq \frac{1}{n}\left(\frac{\mu_{n}}{4}\right)^{s}
$$

so that $\mu_{n} \leqq 4\left(n G_{n s}\right)^{1 / s}$. If $\mu_{n}<4 M$, this inequality in itself serves to give an upper bound for $\mu_{n}$. In any case $\mu_{n}$ can not exceed the sum of the two alternative upper bounds:

$$
\left|T_{n}(x)\right| \leqq \mu_{n} \leqq 4\left(n G_{n s}\right)^{1 / s}+4 M \text {. }
$$

Combination of this relation with the fact that $|f(x)| \leqq M$ gives the conclusion of the lemma.

If $\sigma>s$, it is seen by application of the lemma that 


$$
G_{n \sigma} \leqq\left[4\left(n G_{n s}\right)^{1 / s}+5 M\right]^{\sigma-s} G_{n s} .
$$

Hence

$$
\begin{aligned}
& \left(G_{n \sigma}\right)^{1 /(\sigma-s)} \leqq\left[4\left(n G_{n s}\right)^{1 / s}+5 M\right]\left(G_{n s}\right)^{1 /(\sigma-s)} \\
& \quad=4\left[n^{1-(s / \sigma)} G_{n s}\right]^{\sigma /[s(\sigma-s)]}+5 M\left(G_{n s}\right)^{1 /(\sigma-s)} .
\end{aligned}
$$

If the given sums $T_{n}(x)$ are such that $n^{1-(s / \sigma)} G_{n s}$ approaches zero, $G_{n s}$ by itself will approach zero a fortiori, the whole righthand member will converge toward zero, and the same will be true of $G_{n \sigma}$. For convergence of $G_{n \sigma}$ toward zero as $n$ becomes infinite, when $\sigma>s$, it is sufficient that

$$
\lim _{n \rightarrow \infty} n^{1-(s / \sigma)} G_{n s}=0 .
$$

Although it has not been required in the preceding general argument that the sums $T_{n}(x)$ possess any minimizing property, the reasoning applies in particular if $T_{n}(x)$ is characterized among all trigonometric sums of the $n$th order as one for which $G_{n s}$ has the smallest possible value.

For example, if $T_{n}(x)$ is the partial sum of the Fourier series for $f(x)$ through the terms in $\cos n x$ and $\sin n x$, and if $f(x)$ is of limited variation, $\lim _{n \rightarrow \infty} G_{n \sigma}=0$ for every positive value of $\sigma$. For

$$
G_{n 2}=\pi \sum_{k=n+1}^{\infty}\left(a_{k}^{2}+b_{k}^{2}\right),
$$

where $a_{k}$ and $b_{k}$ are the Fourier coefficients of $f(x)$; these coefficients have an upper bound of the order of $1 / k$; and $G_{n 2}$ consequently has an upper bound of the order of $1 / n$. The same conclusion with regard to the convergence of $G_{n \sigma}$ can also be reached in other ways.

As another illustration, let it be supposed that $f(x)$ is continuous, and that a trigonometric sum $t_{n}(x)$ of the $n$th order exists so that $\left|f(x)-t_{n}(x)\right| \leqq \epsilon_{n}$ for all values of $x$. If $T_{n}(x)$ is a sum which minimizes $G_{n s}$, then by virtue of this minimizing property

$G_{n s}=\int_{-\pi}^{\pi}\left|f(x)-T_{n}(x)\right|^{8} d x \leqq \int_{-\pi}^{\pi}\left|f(x)-t_{n}(x)\right|^{8} d x \leqq 2 \pi \epsilon_{n}{ }^{8}$.

A sufficient condition for the convergence of $G_{n \sigma}$ toward zero as $n$ becomes infinite, when $s$ and $\sigma$ are held fast and the sums 
$T_{n}(x)$ are chosen each time to minimize $G_{n s}$, is that sums $t_{n}(x)$ exist for the successive values of $n$ so that

$$
\lim _{n \rightarrow \infty} n^{1-(s / \sigma)} \epsilon_{n}^{s}=0, \quad \lim _{n \rightarrow \infty} n^{(1 / s)-(1 / \sigma)} \epsilon_{n}=0 .
$$

This condition will be fulfilled, ${ }^{*}$ when $(1 / s)-(1 / \sigma)<1$, if $f(x)$ has a modulus of continuity $\omega(\delta)$ such that

$$
\lim _{\delta \rightarrow 0} \omega(\delta) / \delta^{(1 / s)-(1 / \sigma)}=0 .
$$

The case of Fourier series is included for $s=2$. This special case, however, can be treated more advantageously by a different method. If $T_{n}(x)$ is the partial sum of the Fourier series, and if a trigonometric sum $t_{n}(x)$ of the $n$th order exists with $\epsilon_{n}$ as an upper bound for the difference $\left|f(x)-t_{n}(x)\right|$, then $\dagger$

$$
\left|f(x)-T_{n}(x)\right| \leqq B \epsilon_{n} \log n
$$

for all values of $x$, where $B$ is an absolute constant. By the leastsquare property of the Fourier series,

$$
G_{n 2} \leqq \int_{-\pi}^{\pi}\left[f(x)-t_{n}(x)\right]^{2} d x \leqq 2 \pi \epsilon_{n}^{2}
$$

and for $\sigma>2$

$$
G_{n \sigma} \leqq\left(B \epsilon_{n} \log n\right)^{\sigma-2} G_{n 2} \leqq 2 \pi B^{\sigma-2}(\log n)^{\sigma-2} \epsilon_{n}{ }^{\sigma},
$$

so that $G_{n \sigma}$ will approach zero if $\lim _{n \rightarrow \infty}(\log n)^{1-(2 / \sigma)} \boldsymbol{\epsilon}_{n}=0$, and hence if

$$
\lim _{\delta \rightarrow 0}(\log \delta)^{1-(2 / \sigma)} \omega(\delta)=0,
$$

where $\omega(\delta)$ is the modulus of continuity of $f(x)$.

For general $s$ and for $(1 / s)-(1 / \sigma) \geqq 1$, sufficient conditions for convergence can be formulated in terms of the existence and properties of continuity of one or more derivatives $\ddagger$ of $f(x)$.

* See for example the writer's Theory of Approximation, Colloquium Publications of this Society, vol. 11, 1930, p. 7, Theorem 2.

$\dagger$ See for example the Colloquium cited in the preceding footnote, p. 21, Theorem 9; H. Lebesgue, Sur la représentation trigonométrique approchée des fonctions satisfaisant à une condition de Lipschitz, Bulletin de la Société Mathématique de France, vol. 38 (1910), pp. 184-210; pp. 196-197, 201.

$\ddagger$ See Colloquium, p. 12, Theorem 4. 
Conditions for the convergence of $G_{n \sigma}$ in terms of the order of magnitude of $\epsilon_{n}$ can be stated without change of form if $T_{n}(x)$, instead of minimizing $G_{n s}$, is chosen so as to reduce the integral

$$
\int_{-\pi}^{\pi} \rho(x)\left|f(x)-T_{n}(x)\right|^{8} d x
$$

to a minimum, where $\rho(x)$ is a summable function having a positive lower bound. Similar statements, with a modification of the exponent of the power of $n$, can be made in certain cases when the greatest lower bound of $\rho(x)$ is zero.*

These conclusions relating specifically to sums $T_{n}(x)$ which possess a minimizing property are almost immediate consequences of results previously published as to the order of magnitude of $f(x)-T_{n}(x)$, and have the force of corollaries, pointing out implications already present in those results.

4. Lemma for the Case of Polynomial Approximation. Explicit treatment of polynomial approximation will be confined here to the formulation of an analog of Lemma C.

LEMмA D. If $f(x)$ is a bounded and integrable function over the interval $(a, b)$, with $M$ as an upper bound for its absolute value, if $P_{n}(x)$ is a polynomial of the nth degree, and if

$$
G_{n s}=\int_{a}^{b}\left|f(x)-P_{n}(x)\right|^{8} d x
$$

then

$$
\left|f(x)-P_{n}(x)\right| \leqq 4\left(\frac{4 n^{2} G_{n s}}{b-a}\right)^{1 / s}+5 M
$$

for $a \leqq x \leqq b$.

The proof is similar to that of Lemma C, with use of Markoff's theorem in place of that of Bernstein.

The University of Minnesota

\footnotetext{
* See this Bulletin, loc. cit., Theorems 9 and 10.
} 